Development of a Drive Cycle Simulation Model for Hybrid Powertrain

for parameter estimation and optimization

\author{
Johan Vingbäck
}

Luleå University of Technology 


\section{My research}

- Driveability assessment

- Vehicle dynamics

- Powertrain simulation

- Motion simulators

- Working closely with

- Wintertesting industry

- Automotive industry 


\section{Wintertest industry}

- Started early 70's

- ABS-testing

- Snow intrusion

- Today

- Durability / Quality

- Active safety

- Overall vehicle performance in cold climate 


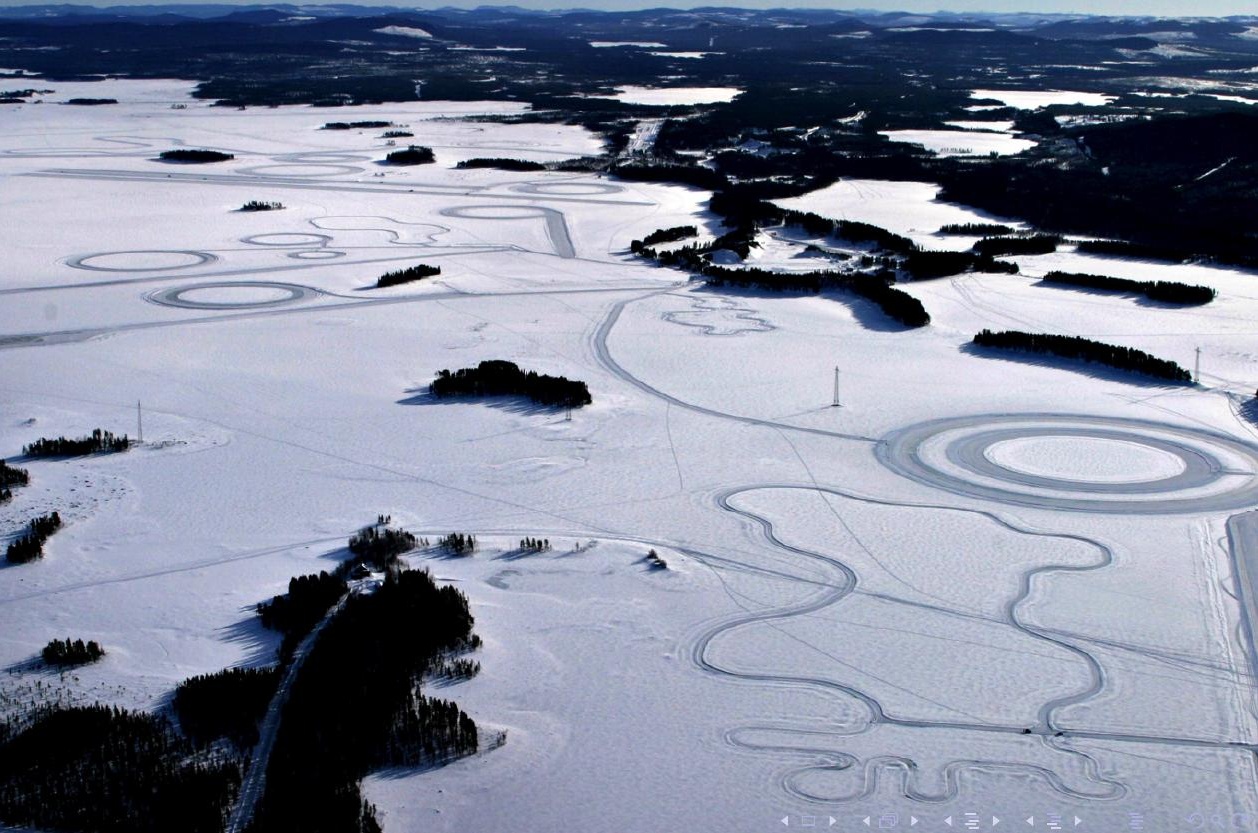




\section{Need}

We need a modular and extendable simulation system for hybrid powertrains.

- The existing simulators for powertrain applications are either too simple or too complex.

- They do not permit rapid and simple modifications to do a parametric study. 


\section{Need}

Therefore, a modular and extendable simulation model was developed.

- Initially

- Standard formulas

- Tabular values, etc

- Develops over time

- More precise formulas

- Measurments

- Co-simulation

- Hardware-in-the-Loop (HIL), etc 


\section{Early development}

- The simulation model was developed in Simulink

- Standard tool

- Graphical development environment

- Extendable for Co-simulation and Hardware-in-the-loop (HIL)

- The degree of abstraction was intended to be high in order to

- Allow rapid parameter changes

- allow high modularity 


\section{Simulink}

- Separation of components is difficult

- Information flow between components is one-directional

- Risk to connect wrong signals

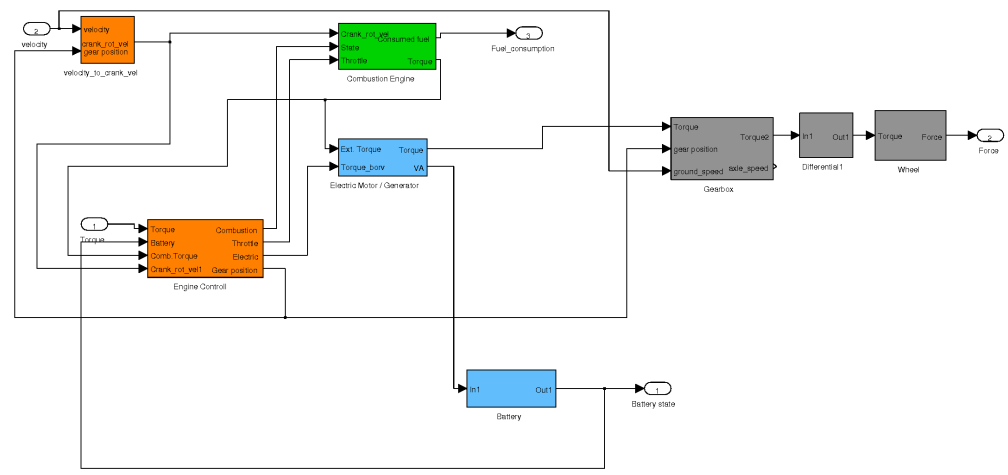




\section{Simscape}

- Components are modelled using "Physical modelling"

- Separation of components is easy

- Information flow is bidirectional

- Information signals is contained in a physical domain 


\section{Simscape}

- The simulation systems is modular

- The physical properties of each component is contained in its corresponding module

- Modules can easily be replaced or extended/modified

- Nodes can only be connected to other nodes within the same physical domain

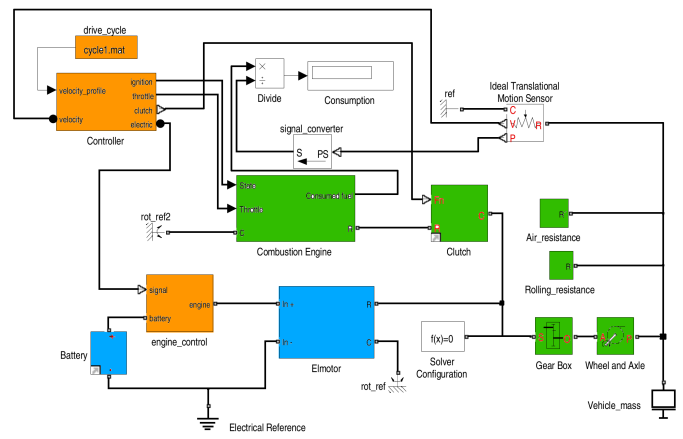




\section{Results}

- The resulting simulation model benefits from the bidirectional information flow of Simscape in multiple disciplines

- Easy to perform parameter studies

- The model is adaptable for almost any driveline setup

- The model is easy to use and extend

- Execution speed is reasonable 


\section{Parameter study \\ "Fuel consumption"}

- Varying combustion engine volume and battery capacity in a series hybrid vehicle

- The combustion engine charged the battery to full capacity after the run

- The simulations shows dependecy between fuel consumption and the two abstract parameters

- Combustion engine volume

- Battery capacity 


\section{Parameter study}

\section{"Fuel consumption"}

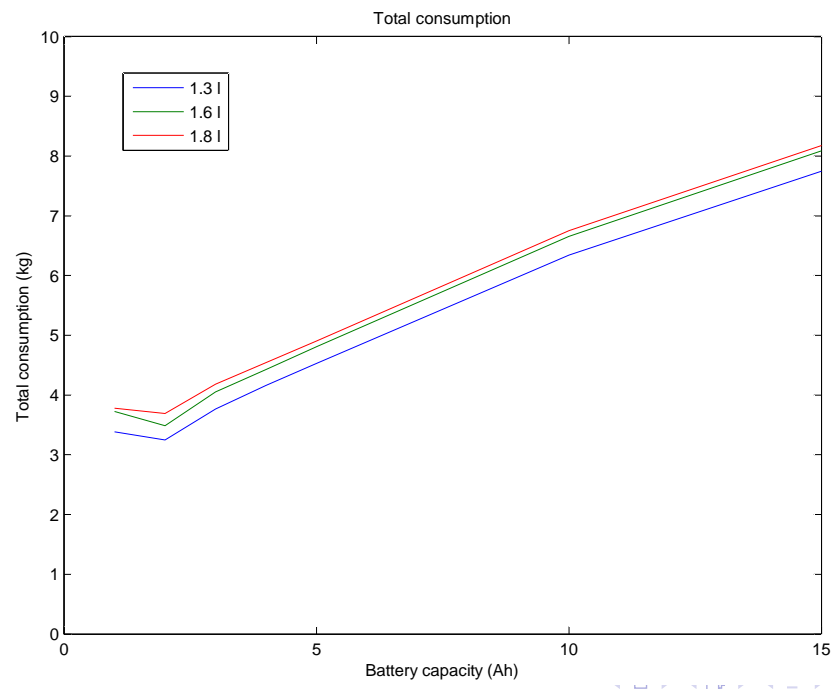




\section{HIL}

- Complex systems with interaction with hardware and software can be challenging to simulate.

- By replacing modules with hardware input, model accuracy may improve, and provide opportunities to test with hardware components in a controlled environment.

- Initial tests include using an Atmel microcontroller connected to a serial port. 
Thank you 